\title{
Non-linear control of variable-speed wind turbines with permanent magnet synchronous generators: a robust backstepping approach
}

\author{
Murat Şeker ${ }^{\mathrm{a}}$, Erkan Zergeroğlu ${ }^{\mathrm{a}, *}$ and Enver Tatlicioğlu ${ }^{\mathrm{b}}$ \\ ${ }^{a}$ Department of Computer Engineering, Gebze Institute of Technology, Gebze, Turkey; ${ }^{b}$ Department of Electrical and Electronics \\ Engineering, Izmir Institute of Technology, Izmir, Turkey
}

(Received 20 February 2013; final version received 5 July 2013)

\begin{abstract}
In this study, a robust backstepping approach for the control problem of the variable-speed wind turbine with a permanent magnet synchronous generator is presented. Specifically, to overcome the negative effects of parametric uncertainties in both mechanical and electrical subsystems, a robust controller with a differentiable compensation term is proposed. The proposed methodology ensures the generator velocity tracking error to uniformly approach a small bound where practical tracking is achieved. Stability of the overall system is ensured by Lyapunov-based arguments. Comparative simulation studies with a standard proportional-integral-type controller are performed to illustrate the effectiveness, feasibility and efficiency of the proposed controller.
\end{abstract}

Keywords: variable-speed wind turbines; non-linear robust control; robust backstepping; power generation from wind

\section{Introduction}

The modern way of living enforces the consumption of electrical energy to grow on a daily basis. Traditional power generation technologies, such as using fossil fuel or nuclear fusion to generate electrical energy, bring about environmental problems, making renewable energy conversion methods more and more popular each day. When compared to the other sources of electrical energy generation, wind is attractive in terms of cost and energy security as it is free and plentiful in supply. Partly due to this, electrical energy generation from wind power using wind turbines is a growing sector.

A significant amount of scientific research has been conducted on the control of wind turbine systems, especially utilising sliding-mode control (Beltran, AhmedAli, and Hachemi Benbouzid 2008), proportional-integralderivative (PID) (Abdin and $\mathrm{Xu} 2000$; Maureen and Balas 2002), fuzzy-adaptive control (Galdi, Piccolo, and Siano 2008), non-linear robust control (Iyasere, Salah, Dawson, and Wagner 2008) and even wind-model-based predictive control (Senjyu et al. 2006). In Beltran et al. (2008), authors proposed a control strategy that is based on a sliding-mode controller to maximise the power efficiency where the turbine tip speed ratio must be maintained at its optimum value despite wind variations. In Iyasere et al. (2008), authors rendered their control strategy based on average wind speed and standard deviation of wind speed and used a generalised predictive controller. A PID controller using the pole assignment technique was presented in Maureen and Balas (2002). In Oguz and Guney (2010), authors performed the dynamic modeling and simulation of the wind power generation system and control of blade pitch angle which was validated by a neuro-fuzzy controller and a conventional PID controller separately.

From the review of relevant work in the literature we can conclude that there are two typical ways of operating wind turbines: (1) at a constant speed or (2) at variable speed. Constant-speed wind turbines can be operated by means of active pitch control and/or stall-regulated control, and are typically connected directly to the power grid. However, when the turbine is operated at nearly constant speed, the aerodynamic torque control can be sensitive to gust and might create spikes on the power grid. On the other hand, variable speeds allows the wind turbine to operate at a higher efficiency, thus enabling extraction of more power from the wind. As stated in Müller, Deicke, and De Doncker (2002), variable-speed wind turbines are more advantageous, as they increase energy capture, has the ability to operate at maximum power point, improve efficiency and has power quality. The quality of power generation (and output regulation) strongly depend on the control technique employed (Muldaji, Pierce, and Migliore 1998), that is, the effectiveness of the wind power generation relies mostly on the control strategy. Therefore, for variable-speed wind turbines, the wind power is truly cost effective via the use of advanced control techniques, such as model-based control design approaches. The main disadvantage of model-based control designs is that mechanical and electrical parameter values of wind turbines in most cases are not available. The effects of uncertainties in the turbine dynamics

*Corresponding author. Email: e.zerger@gyte.edu.tr 
become more apparent in practical applications in which the unknown system parameters limit the efficient energy capture of a wind turbine. To overcome the aforementioned problems, a vast number of control strategies have been proposed for variable-speed wind turbines. In Muldaji et al. (1998), authors have evaluated a variable-speed, stall-regulated strategy that eliminates the need for ancillary aerodynamic control systems. In Boukhezzar and Siguerdidjane (2005), a cascade structure, non-linear controller was proposed; however, parametric uncertainties of the system were not taken into account. In Song, Dhinarakaran, and Bao (2000), authors presented two non-linear controllers: (1) an exact model knowledge controller that assumes the system parameters are available and (2) an adaptive controller which compensates for the uncertainties in the mechanical subsystem parameters but requirs exact knowledge of the parameters of the electrical subsystem for the controller implementation. In Ozbay, Zergeroglu, and Sivrioglu (2008) and Sivrioglu, Ozbay, and Zergeroglu (2008), the control design in Song et al. (2000) was extended to compensate for the uncertainties in both electrical and mechanical subsystems. In Sivrioglu et al. (2008), the issues of external disturbances and modeling errors were also addressed. Recently high-order sliding-mode controllers have also been applied to variable-speed wind turbines (Beltran, Ahmed-Ali, and Hachemi Benbouzid 2009; Morfin, Loukianov, Canedo, and Castellanos 2010). In Beltran et al. (2009), authors applied a second-order sliding-mode controller for the power generation control in variable-speed wind turbines. The proposed sliding-mode methodology was effective in terms of power regulation despite the parametric uncertainties in the turbine and the generator. In Morfin et al. (2010), a robust non-linear controller based on block linearisation combined with a second-order slidingmode technique was applied to a wound rotor induction generator.

In this paper, ${ }^{1}$ we have extended the previous results given in Ozbay et al. (2008) and Sivrioglu et al. (2008) to a more sophisticated variable-speed wind turbine model, and present a novel robust backstepping approach for the control problem of the variable-speed wind turbine with a permanent magnet synchronous generator (PMSG). Specifically, to compensate for the undesirable effects of parametric uncertainties, of both mechanical and electrical subsystems, a differentiable robust controller is proposed. Robust backstepping-type controllers for various classes of non-linear systems have been proposed in the control literature ( $\mathrm{Qu}$ 1998). Some of the designs were used to back up neural network (Jagannathan and Lewis 2000) and fuzzy logic-type controllers (Kwan and Lewis 2000). When compared to recently introduced high-order sliding-mode observation techniques presented in Ferreira, Bejarano, and Fridman (2012), robust backstepping-type controllers present smoother controller action and can be applied to a broader class of dynamical systems. The method used in this work relays on a high-gain approach as opposed to high-frequency techniques presented in $\mathrm{Qu}$ (1998) and Dawson, Bridges, and Qu (1995). The proposed methodology ensures the generator velocity tracking error to uniformly approach a small bound where practical tracking is achieved. Simulation studies are presented to illustrate the efficiency and feasibility of the proposed controller.

\section{Dynamical model}

The mathematical equations describing the dynamics of a variable-speed wind turbine with PMSG in $d-q$ coordinates are assumed to have the following forms (Abedini and Nikkhajoei 2011):

$$
\begin{gathered}
J \frac{d \omega_{g}}{d t}=-\frac{1.5 P^{2}}{4} \lambda_{m} i_{d}-\frac{B P}{2} \omega_{g}+\frac{P}{2} T_{m}, \\
L_{d} \frac{d i_{d}}{d t}=-R_{s} i_{d}-L_{q} i_{q} \omega_{g}+k_{g} \lambda_{m} \omega_{g}-v_{d}, \\
L_{q} \frac{d i_{q}}{d t}=L_{d} i_{d} \omega_{g}-R_{s} i_{q}-v_{q},
\end{gathered}
$$

where the first equation is for the mechanical subsystem and the last two equations represent electrical subsystem dynamics. Specifically, $v_{d}(t), v_{q}(t) \in \mathbb{R}$, and $i_{d}(t), i_{q}(t) \in$ $\mathbb{R}$ are the general $d-q$ terminal voltages and currents, respectively, $R_{s} \in \mathbb{R}$ is the stator resistance, $L_{d}, L_{q} \in \mathbb{R}$ are the $d-$ and $q$-axis inductances, respectively; $\lambda_{m} \in$ $\mathbb{R}$ is the permanent magnet flux, $k_{g} \in \mathbb{R}$ is the generator coefficient, $T_{m}(t) \in \mathbb{R}$ is the input mechanical torque of the wind turbine, $\omega_{g}(t) \in \mathbb{R}$ is the generator velocity, $P \in \mathbb{R}$ is the number of poles, $J \in \mathbb{R}$ is the rotor moment of inertia and $B \in \mathbb{R}$ is the friction and windage coefficient. Terminal voltages $v_{d}(t)$ and $v_{q}(t)$ are the control input signals in Equations (1)-(3).

A schematic representation of the system under consideration is presented in Figure 1. The main control objective is to ensure that the generator velocity, $\omega_{g}(t)$, tracks a smooth reference velocity profile denoted by $\omega_{d}(t) \in \mathbb{R}$ and generates according to the operational modes of the wind turbine, despite the lack of exact knowledge of both mechanical and electrical subsystem parameters of Equations (1)-(3). The control objective will be achieved by properly designing the controller system block given in Figure 1.

\section{Error system development}

To quantify the control objective, we define error signals, denoted by $e(t), z_{1}(t), z_{2}(t) \in \mathbb{R}$, as follows:

$$
\begin{gathered}
e \triangleq \omega_{d}-\omega_{g}, \\
z_{1} \triangleq i_{d, \text { ref }}-i_{d}, \\
z_{2} \triangleq i_{q, \text { ref }}-i_{q},
\end{gathered}
$$




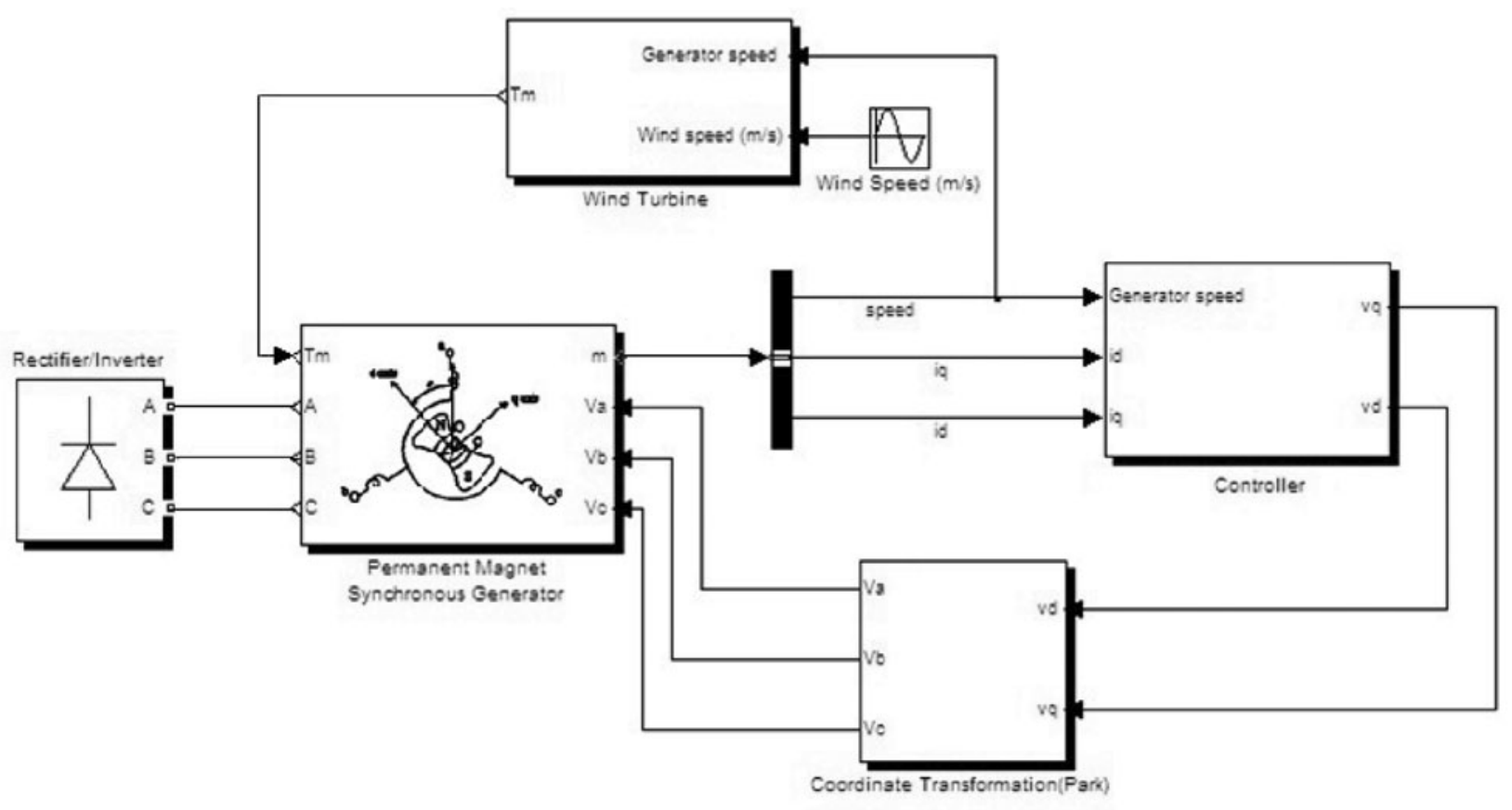

Figure 1. Overall system.

where $i_{d, \text { ref }}(t), i_{q, \text { ref }}(t) \in \mathbb{R}$ are reference signals that will be designed subsequently. After taking the time derivative of the tracking error term in Equation (4), pre-multiplying with $J$, and then utilising Equation (1), we obtain

$$
J \dot{e}=J \dot{\omega}_{d}+\frac{B P}{2} \omega_{g}+\frac{1.5 P^{2}}{4} \lambda_{m} i_{d}-\frac{P}{2} T_{m} .
$$

The above expression can be rewritten in the following simpler form:

$$
J \dot{e}=Y \phi+f+\phi_{m} i_{d},
$$

where $f(t) \triangleq-\frac{P}{2} T_{m} \in \mathbb{R}$ contains smooth time-varying uncertainties, $\phi_{m} \triangleq \frac{1.5 P^{2}}{4} \lambda_{m} \in \mathbb{R}$ is an uncertain constant parameter, $Y(t) \triangleq\left[\dot{\omega}_{d} \omega_{g}\right] \in \mathbb{R}^{1 \times 2}$ contains time-varying functions and $\phi \triangleq\left[J \frac{B P}{2}\right]^{T} \in \mathbb{R}^{2}$ contains uncertain constant parameters. Based on the definition of $Y(\cdot)$, its desired form is defined as $Y_{d}(t) \triangleq\left[\dot{\omega}_{d} \omega_{d}\right] \in \mathbb{R}^{1 \times 2}$. After adding and subtracting $Y_{d} \phi$ to the right-hand side of the open-loop mechanical subsystem dynamics, the following expression can be obtained:

$$
J \dot{e}=F+\chi+\widetilde{\phi}_{m} i_{d}+\hat{\phi}_{m} i_{d, \text { ref }}-\hat{\phi}_{m} z_{1},
$$

where $\hat{\phi}_{m} \in \mathbb{R}$ is the best-guess estimate (nominal value) of $\phi_{m}, \widetilde{\phi}_{m} \triangleq \phi_{m}-\hat{\phi}_{m} \in \mathbb{R}$ is the estimation error and $F(t)$, $\chi(t) \in \mathbb{R}$ are defined as

$$
F \triangleq Y_{d} \phi+f
$$

$$
\chi \triangleq\left(Y-Y_{d}\right) \phi .
$$

Based on the subsequent stability analysis, reference signals $i_{d, \text { ref }}(t)$ and $i_{q, \text { ref }}(t)$ are designed as

$$
\begin{gathered}
i_{d, \mathrm{ref}}=\frac{-1}{\hat{\phi}_{m}}\left\{Y_{d} \hat{\phi}+\hat{f}+\left(k_{e}+k_{n} \rho_{1}^{2}\right) e+v_{R 1}\right\}, \\
i_{q, \mathrm{ref}}=0,
\end{gathered}
$$

where $k_{e}, k_{n} \in \mathbb{R}$ are positive control gains, $\hat{\phi}, \hat{\phi}_{m}, \hat{f} \in$ $\mathbb{R}$ are the constant best-guess estimates of the unknown parameters $\phi, \phi_{m}$ and $f$, respectively; the positive bounding function $\rho_{1}(e) \in \mathbb{R}$ is designed to satisfy

$$
\rho_{1} \geq|\chi|
$$

and $v_{R 1}(t) \in \mathbb{R}$ is a robust term designed in the following form:

$$
v_{R 1}=\frac{e \rho_{2}^{2}(|e|)}{\epsilon_{1}}
$$

where $\epsilon_{1} \in \mathbb{R}$ is a positive constant and $\rho_{2}(|e|) \in \mathbb{R}$ is a positive bounding function that is designed to satisfy

$$
\rho_{2}(|e|) \geq|\widetilde{F}|,
$$

where $\widetilde{F}(t) \triangleq F-Y_{d} \hat{\phi}-\hat{f} \in \mathbb{R}$. 
Remark 1: Note that the robust controller design enforces the use of the values of the upper bounds of the uncertain functions in the controller design, as $\rho_{2}(|e|)$ of Equation (16). In this work, it is assumed that for each bounding function, say, $\rho_{i}$, defined such that $\rho_{i}>|f(t)|$, with the function $f(t)$ containing some uncertain parameters, a rough estimate of the upper and lower bounds of the function containing the uncertainties is available. Choosing the values of these upper bounding functions extremely high to ensure the validity of the inequalities might seem to be proper at first. However, this would end up with unnecessary high gains which in turn would result in high control actions and in some cases even instability.

Remark 2: The backstepping procedure requires that the auxiliary control in Equation (12) be differentiable; hence, the high-gain robust control term in Equation (15) was defined to ensure differentiability. The design of a differentiable high-frequency robust term is also possible as illustrated in Seker et al. (2012). However, our experience with the simulation studies showed that using a high-gain robust term as opposed to a high-frequency robust term reduced the chattering in the control signal for this particular application.

After inserting $i_{d, \text { ref }}(t)$ back into the open-loop mechanical subsystem dynamics, the following expression is obtained:

$J \dot{e}=-k_{e} e-k_{n} \rho_{1}^{2} e+\chi-\hat{\phi}_{m} z_{1}+\widetilde{F}+\widetilde{\phi}_{m} i_{d}-v_{R 1}$.

Note that the term $\widetilde{\phi}_{m} i_{d}$ can be upper bounded as

$$
\rho_{3}\left(\left|z_{1}\right|\right) \geq\left|\widetilde{\phi}_{m} i_{d}\right|,
$$

where $\rho_{3}\left(\left|z_{1}\right|\right) \in \mathbb{R}$ is a positive bounding function. The backstepping design also requires the dynamics of the auxiliary error signals $z_{1}(t)$ and $z_{2}(t)$. To obtain the dynamics for $z_{1}(t)$, first the derivative of Equation (5) is taken, and then the time derivative of Equation (12) is inserted and the resulting expression is multiplied with $L_{d}$ to obtain

$$
\begin{aligned}
L_{d} \dot{z}_{1}= & \frac{-L_{d}}{\hat{\phi}_{m}}\left\{\dot{Y}_{d} \hat{\phi}+\frac{1}{J}\left(k_{e}+2 k_{n} \rho_{1} e \frac{\partial}{\partial e} \rho_{1}\right.\right. \\
& \left.\left.+k_{n} \rho_{1}^{2}+\frac{\partial}{\partial e} v_{R 1}\right) \times\left(Y \phi+f+\phi_{m} i_{d}\right)\right\} \\
& +R_{s} i_{d}+L_{q} i_{q} \omega_{g}+v_{d}-k_{g} \lambda_{m} \omega_{g},
\end{aligned}
$$

which can be rewritten in the following manner:

$$
L_{d} \dot{z}_{1}=W_{1} \theta_{1}+f_{1}+v_{d},
$$

where $W_{1}(\cdot) \theta_{1} \in \mathbb{R}$ contains the linearly parameterisable terms with $W_{1}(t) \in \mathbb{R}^{1 \times 8}$ and $\theta_{1} \in \mathbb{R}^{8}$ containing time- varying functions and uncertain constant parameters (detailed definitions are given in Appendix A), respectively, and $f_{1}(t) \in \mathbb{R}$ contains the time-varying uncertainties, which are explicitly defined as follows:

$$
\begin{array}{r}
W_{1} \theta_{1} \triangleq \\
\frac{-L_{d}}{\hat{\phi}_{m}}\left\{\dot{Y}_{d} \hat{\phi}+\frac{1}{J}\left(k_{e}+2 k_{n} \rho_{1} e \frac{\partial}{\partial e} \rho_{1}\right.\right. \\
\left.\left.+k_{n} \rho_{1}^{2}+\frac{\partial}{\partial e} v_{R 1}\right) \times\left(Y \phi+\phi_{m} i_{d}\right)\right\} \\
+R_{s} i_{d}+L_{q} i_{q} \omega_{g}-k_{g} \lambda_{m} \omega_{g} \\
f_{1} \triangleq \frac{-L_{d}}{\hat{\phi}_{m}} \frac{1}{J}\left(k_{e}+2 k_{n} \rho_{1} e \frac{\partial}{\partial e} \rho_{1}+k_{n} \rho_{1}^{2}+\frac{\partial}{\partial e} v_{R 1}\right) f .
\end{array}
$$

Based on the subsequent stability analysis, $v_{d}(t)$ is designed in the following form:

$$
v_{d}=-\left(k_{1}+k_{n} \rho_{3}^{2}\right) z_{1}-W_{1} \hat{\theta}_{1}+\hat{\phi}_{m} e-v_{R 2},
$$

where $\hat{\theta}_{1} \in \mathbb{R}^{8}$ is the constant best-guess estimate of the unknown parameter vector $\theta_{1}, v_{R 2}(t) \in \mathbb{R}$ is an additional robust control term, $k_{1} \in \mathbb{R}$ is a positive constant control gain and $\rho_{3}(\cdot)$ was introduced in Equation (18). In Equation (23), the robust term $v_{R 2}(t)$ is introduced to compensate for the mismatch between the actual and estimated parameters, and is explicitly designed as follows:

$$
v_{R 2}=\frac{z_{1} \rho_{4}^{2}}{\epsilon_{2}},
$$

where $\epsilon_{2} \in \mathbb{R}$ is a positive constant, and $\rho_{4}(t) \in \mathbb{R}$ is a positive bounding function designed to satisfy

$$
\rho_{4} \geq\left|W_{1} \tilde{\theta}_{1}\right|+\left|f_{1}\right|,
$$

where $\widetilde{\theta}_{1} \triangleq \theta_{1}-\hat{\theta}_{1} \in \mathbb{R}^{8}$ is the parameter estimation error. After substituting Equation (23) into Equation (20), the closed-loop dynamics for $z_{1}(t)$ is obtained to have the following form:

$$
L_{d} \dot{z}_{1}=-k_{1} z_{1}-k_{n} \rho_{3}^{2} z_{1}+W_{1} \tilde{\theta}_{1}+f_{1}+\hat{\phi}_{m} e-v_{R 2} .
$$

Similarly, the dynamics of $z_{2}(t)$ can be obtained to have the following form:

$$
L_{q} \dot{z}_{2}=-L_{d} i_{d} \omega_{g}+R_{s} i_{q}+v_{q}
$$

After defining $W_{2}(t) \triangleq\left[-i_{d} \omega_{g} i_{q}\right] \in \mathbb{R}^{1 \times 2}$, which contains time-varying functions, and $\theta_{2} \triangleq\left[\begin{array}{ll}L_{d} R_{s}\end{array}\right]^{T} \in$ $\mathbb{R}^{2}$, which contains uncertain constant parameters, the right-hand side of Equation (27) can be rewritten as

$$
L_{q} \dot{z}_{2}=W_{2} \theta_{2}+v_{q}
$$


Similar to the design of $v_{d}(t)$, the voltage input $v_{q}(t)$ is designed in the following form:

$$
v_{q}=-k_{2} z_{2}-W_{2} \hat{\theta}_{2}-v_{R 3},
$$

where $k_{2} \in \mathbb{R}$ is a positive constant control gain, $\hat{\theta}_{2} \in \mathbb{R}^{2}$ is the constant best-guess estimate of the unknown parameter vector $\theta_{2}$ and $v_{R 3}(t) \in \mathbb{R}$ is an additional robust control term introduced to compensate for the mismatch between the actual and estimated parameters, which is explicitly defined as follows:

$$
v_{R 3}=\frac{z_{2} \rho_{5}^{2}}{\epsilon_{3}},
$$

where $\epsilon_{3} \in \mathbb{R}$ is a positive constant, and $\rho_{5}(t) \in \mathbb{R}$ is a positive bounding function that is designed to satisfy

$$
\rho_{5} \geq\left|W_{2} \tilde{\theta}_{2}\right|
$$

where $\widetilde{\theta}_{2} \triangleq \theta_{2}-\hat{\theta}_{2} \in \mathbb{R}^{2}$ is the parameter estimation error. Substituting Equation (29) into Equation (28), the closedloop dynamics for $z_{2}(t)$ is obtained to have the following form:

$$
L_{q} \dot{z}_{2}=-k_{2} z_{2}+W_{2} \tilde{\theta}_{2}-v_{R 3}
$$

\section{Stability analysis}

After obtaining the closed-loop error dynamics for the signals $e(t), z_{1}(t)$ and $z_{2}(t)$, the following theorem can now be stated.

Theorem 4.1: The robust controller given by Equations (23) and (29) and the auxiliary control inputs (12) and (13) with the robust terms (15), (24) and (30) guarantee uniformly ultimate boundedness of the generator velocity tracking error signal e $(t)$ in the sense that

$$
\|e(t)\| \leq \sqrt{\frac{a}{b}\|x(0)\|^{2} \exp (-\beta t)+\frac{2 \epsilon}{b \beta}(1-\exp (-\beta t))},
$$

where $x \triangleq\left[\begin{array}{lll}e & z_{1} & z_{2}\end{array}\right]^{T} \in \mathbb{R}^{3}$ is the combined error signal and $a, b, \beta, \epsilon \in \mathbb{R}$ are positive constants defined as

$$
\begin{gathered}
a \triangleq \max \left\{J, L_{d}, L_{q}\right\}, \\
b \triangleq \min \left\{J, L_{d}, L_{q}\right\}, \\
\beta \triangleq \frac{2 \min \left\{\left(k_{e}-\frac{1}{4 k_{n}}\right), k_{1}, k_{2}\right\}}{\max \left\{J, L_{d}, L_{q}\right\}}, \\
\epsilon \triangleq \epsilon_{1}+\epsilon_{2}+\epsilon_{3}+\frac{1}{4 k_{n}},
\end{gathered}
$$

where $\epsilon_{1}, \epsilon_{2}, \epsilon_{3}, k_{e}, k_{1}, k_{2}$ and $k_{n}$ were previously defined.
Proof: Consider the following non-negative scalar function,

$$
V \triangleq \frac{1}{2} J e^{2}+\frac{1}{2} L_{d} z_{1}^{2}+\frac{1}{2} L_{q} z_{2}^{2}
$$

which can be lower and upper bounded in the following form:

$$
\frac{1}{2} b\|x\|^{2} \leq V \leq \frac{1}{2} a\|x\|^{2} .
$$

Taking the time derivative of Equation (38) along Equations (17), (26) and (32), and then cancelling the common terms, yields

$$
\begin{aligned}
\dot{V}= & -k_{e} e^{2}-k_{1} z_{1}^{2}-k_{2} z_{2}^{2} \\
& +\left[\chi-k_{n} \rho_{1}^{2} e\right] e \\
& +\left[\widetilde{F}-\frac{e \rho_{2}^{2}(|e|)}{\epsilon_{1}}\right] e \\
& +\left[\widetilde{\phi}_{m} i_{d} e-k_{n} \rho_{3}^{2} z_{1}\right] z_{1} \\
& +\left[W_{1} \widetilde{\theta}_{1}+f_{1}-\frac{z_{1} \rho_{4}^{2}}{\epsilon_{2}}\right] z_{1} \\
& +\left[W_{2} \widetilde{\theta}_{2}-\frac{z_{2} \rho_{5}^{2}}{\epsilon_{3}}\right] z_{2} .
\end{aligned}
$$

After using Equations (14), (16), (18), (25) and (31), the right-hand side of Equation (40) can be upper bounded as follows:

$$
\begin{aligned}
\dot{V} \leq & -k_{e} e^{2}-k_{1} z_{1}^{2}-k_{2} z_{2}^{2} \\
& +\left[\rho_{1}|e|-k_{n} \rho_{1}^{2} e^{2}\right] \\
& +\rho_{2}|e|\left[1-\frac{\rho_{2}|e|}{\epsilon_{1}}\right] \\
& +\left[\rho_{3}\left|z_{1}\right||e|-k_{n} \rho_{3}^{2} z_{1}^{2}\right] \\
& +\rho_{4}\left|z_{1}\right|\left[1-\frac{\rho_{4}\left|z_{1}\right|}{\epsilon_{2}}\right] \\
& +\rho_{5}\left|z_{2}\right|\left[1-\frac{\rho_{5}\left|z_{2}\right|}{\epsilon_{3}}\right] .
\end{aligned}
$$

After adding and subtracting $\frac{1}{4 k_{n}}$ and then completing the squares of the first and third bracketed terms of Equation (41), it can further be upper bounded as

$$
\begin{aligned}
\dot{V} \leq & -\min \left\{\left(k_{e}-\frac{1}{4 k_{n}}\right), k_{1}, k_{2}\right\}\|x\|^{2} \\
& +\rho_{2}|e|\left[1-\frac{\rho_{2}|e|}{\epsilon_{1}}\right] \\
& +\rho_{4}\left|z_{1}\right|\left[1-\frac{\rho_{4}\left|z_{1}\right|}{\epsilon_{2}}\right]
\end{aligned}
$$




$$
+\rho_{5}\left|z_{2}\right|\left[1-\frac{\rho_{5}\left|z_{2}\right|}{\epsilon_{3}}\right]+\frac{1}{4 k_{n}}
$$

where the following was used:

$$
\rho_{3}\left|z_{1}\right||e|-k_{n} \rho_{3}^{2} z_{1}^{2} \leq \frac{e^{2}}{4 k_{n}}
$$

Finally, upper bounding the terms in the brackets allows us to place an upper bound on the right-hand side of Equation (42) as shown below,

$$
\dot{V} \leq-\min \left\{\left(k_{e}-\frac{1}{4 k_{n}}\right), k_{1}, k_{2}\right\}\|x\|^{2}+\epsilon,
$$

where the bounding property,

$$
\rho_{i+2}\left|z_{i-1}\right|\left[1-\frac{\rho_{i+2}\left|z_{i-1}\right|}{\epsilon_{i}}\right]<\epsilon_{i}
$$

with $i=1,2,3$ and $z_{0}=e$, has been utilised and $\epsilon$ was previously defined in Equation (37). From the upper bound on $v(t)$ given in Equation (39), the upper bound $\dot{V}(t)$ in Equation (44) can now be shown as

$$
\dot{V} \leq-\beta V+\epsilon,
$$

where $\beta$ was previously defined in Equation (36). Notice that, to ensure the positiveness of $\beta$, the control gain $k_{n}$ has to be selected to ensure $k_{n}>\frac{1}{4 k_{e}}$. The differential inequality of Equation (46) can now be solved to yield (Dawson et al. 1995)

$$
V(t) \leq V(0) \exp (-\beta t)+\frac{\epsilon}{\beta}(1-\exp (-\beta t))
$$

After applying the bounds of Equation (39) to Equation (47), the following upper bound for $x(t)$ is obtained:

$$
\|x(t)\| \leq \sqrt{\frac{a}{b}\|x(0)\|^{2} \exp (-\beta t)+\frac{2 \epsilon}{b \beta}(1-\exp (-\beta t))},
$$

where $a$ and $b$ were previously defined in Equations (34) and (35), respectively. Based on Equation (48) and the definition of $x(t)$, the generator velocity tracking error $e(t)$ can be shown to be bounded as given by Equation (33) (Dawson, $\mathrm{Hu}$, and Burg 1998). Due to the boundedness of $e(t), z_{1}(t)$, and $z_{2}(t)$, standard signal chasing arguments can be utilised to show that all the signals in the closed-loop systems in Equations (17), (26) and (32) are bounded.

\section{Simulation results}

To demonstrate the performance of the proposed robust controller, two different simulation studies were performed similar to that of Song et al. (2000). For the first simulation, the reference generator velocity $\omega_{d}(t)$ was selected as

$$
\omega_{d}(t)=2+\sin (t)
$$

and for the second one, a more realistic reference generator velocity was selected as

$$
\omega_{d}(t)= \begin{cases}0, & u(k)<u_{c}, \\ x_{m}\left(1+\sin \left(\frac{\pi}{2} \frac{\left(u(k)-s_{1}\right)}{d_{1}}\right)\right), & u(k)<u_{r}, \\ x_{m}, & u(k)<u_{F}, \\ x_{m}\left(1+\sin \left(\frac{\pi}{2} \frac{\left(u(k)-s_{2}\right)}{d_{2}}\right)\right), & u(k)<u_{s}, \\ 0, & u(k)>u_{s},\end{cases}
$$

where $u(k)$ is an auxiliary function used in the desired trajectory generation with

$$
\begin{gathered}
s_{1}=\frac{1}{2}\left(u_{r}+u_{c}\right), d_{1}=\frac{1}{2}\left(u_{r}-u_{c}\right), \\
s_{2}=\frac{1}{2}\left(u_{r}+u_{F}\right), d_{2}=\frac{1}{2}\left(u_{r}-u_{F}\right), \\
u_{s}=36.3[\mathrm{~m} / \mathrm{sec}], x_{m}=4.1[\mathrm{rad} / \mathrm{sec}], \\
u_{c}=9.3[\mathrm{~m} / \mathrm{sec}], u_{r}=12.7[\mathrm{~m} / \mathrm{sec}], \\
u_{F}=32.9[\mathrm{~m} / \mathrm{sec}],
\end{gathered}
$$

where the parameter $x_{m}$ was specified according to the allowable generator velocity.

Simulations were performed on a Matlab/Simulink simulation platform on a standard PC. The controller algorithms were implemented using C-Mex S-function blocks and the control frequency was selected as $1 \mathrm{KHz}$. A block diagram representation of the simulations are given in Figure 2. The system parameters used in the simulations were

$$
\begin{aligned}
L_{d} & =0.002, L_{q}=0.002, R_{s}=0.18, k_{g}=100, \\
J & =0.48, B=0.001, \lambda=0.8, p=8 .
\end{aligned}
$$

To compare the performance of the proposed robust method with the performance of a standard controller, two simulations on a proportional-integral-type (PI-type) controller of the following form were also performed:

$$
\begin{aligned}
i_{d, \mathrm{ref}} & =k_{P, e} e+k_{I, e} \int_{0}^{t} e(\sigma) d \sigma, \\
v_{d} & =k_{P, z_{1}} z_{1}+k_{I, z_{1}} \int_{0}^{t} z_{1}(\sigma) d \sigma-w_{g} L_{d} i_{d, \text { ref }}, \\
v_{q} & =k_{P, z_{2}} z_{2}+k_{I, z_{2}} \int_{0}^{t} z_{2}(\sigma) d \sigma+w_{g}\left(L_{q} i_{q, \mathrm{ref}}+\lambda_{m}\right),
\end{aligned}
$$

where the gains (i.e., $k_{P, e}, k_{I, e}, k_{P, z_{1}}, k_{I, z_{1}}, k_{P, z_{2}}, k_{I, z_{2}} \in$ $\mathbb{R})$ were tuned until the best tracking performance was 


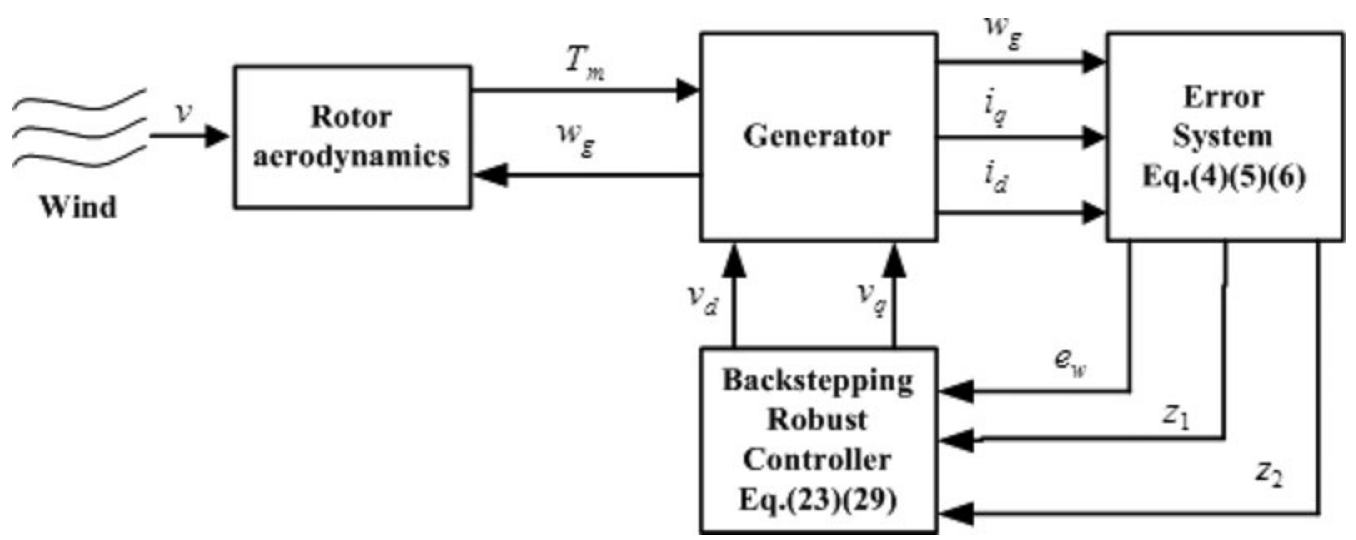

Figure 2. Block diagram representation of controller implementation.

obtained. The controller gains were calculated as

$$
\begin{gathered}
k_{P, e}=571.1, k_{I, e}=0.46 \\
k_{P, z_{1}}=184.0164, k_{I, z_{1}}=0.0002, \\
k_{P, z_{2}}=36.515, k_{I, z_{2}}=0.005236 .
\end{gathered}
$$

It is important to highlight that the implementation of the PI-type controller required the knowledge of the system parameters $L_{d}, L_{q}$ and $\lambda$.

Similar to the PI-type controller, the controller gains of the proposed robust method were tuned until the best tracking performance was obtained and the controller gains were chosen as

$$
\begin{aligned}
& k_{e}=3, k_{n}=9, k_{1}=1, k_{2}=35, \\
& \rho_{1}=1.6, \rho_{2}=1, \rho_{3}=14, \rho_{4}=30, \rho_{5}=10, \\
& \varepsilon_{1}=0.0004, \varepsilon_{2}=0.1, \varepsilon_{3}=0.01 .
\end{aligned}
$$

The best-guess estimates of the system parameters were set to $80 \%$ of their actual values.

The results of the first set of simulations (i.e., sinusoidal tracking) are presented through Figures A1-A4. The data obtained from the simulations are then illustrated in Table 1. The desired and actual generator velocity profiles for the PI-type and proposed robust backstepping controllers are given in Figure A1, with the tracking error performances

Table 1. The results of the first simulation.

\begin{tabular}{lccccccc}
\hline & \multicolumn{3}{c}{ Robust backstepping } & & \multicolumn{3}{c}{ Standard PI type } \\
\cline { 2 - 4 } & $T=12.7 \mathrm{sec}$ & $T=22.9 \mathrm{sec}$ & $T=36 \mathrm{sec}$ & & $T=12.7 \mathrm{sec}$ & $T=22.9 \mathrm{sec}$ & $T=36 \mathrm{sec}$ \\
\hline$|e(T)|$ & 0.015 & 0.05 & 0.05 & & 0.024 & 0.05 & 0.081 \\
$\int_{0}^{T}|e(\sigma)| d \sigma$ & 1.2 & 1.6 & 1.6 & & 2.25 & 2.58 & 3.45 \\
$\left|v_{d}(T)\right|$ & 158 & 165 & 200 & & 275 & 320 & 130 \\
$\int_{0}^{T}\left|v_{d}(\sigma)\right| d \sigma$ & 11,300 & 12,600 & 13,900 & & 70,000 & 71,800 & 73,000 \\
$\left|v_{q}(T)\right|$ & 77.4 & 157.96 & 360 & & 67.5 & 139.5 & 337.1 \\
$\int_{0}^{T}\left|v_{q}(\sigma)\right| d \sigma$ & 460 & 1600 & 4618 & & 394 & 1400 & 4125 \\
\hline
\end{tabular}

Table 2. The results of the second simulation.

\begin{tabular}{lccccccc}
\hline & \multicolumn{3}{c}{ Robust backstepping } & & \multicolumn{3}{c}{ Standard PI type } \\
\cline { 2 - 3 } & $T=12.7 \mathrm{sec}$ & $T=22.9 \mathrm{sec}$ & $T=36 \mathrm{sec}$ & & $T=12.7 \mathrm{sec}$ & $T=22.9 \mathrm{sec}$ & $T=36 \mathrm{sec}$ \\
\hline$|e(T)|$ & 0.013 & 0.0031 & 0.05 & & 0.064 & 0.013 & 0.05 \\
$\int_{0}^{T}|e(\sigma)| d \sigma$ & 0.84 & 0.85 & 1.07 & & 0.54 & 0.85 & 1.07 \\
$\left|v_{d}(T)\right|$ & 225 & 14.43 & 3 & & 6680 & 1500 & 4838 \\
$\int_{0}^{T}\left|v_{d}(\sigma)\right| d \sigma$ & 4967 & 4784 & 5350 & & 48,500 & 81,800 & 10,000 \\
$\left|v_{q}(T)\right|$ & 110 & 157.96 & 360 & & 95.62 & 200 & 360 \\
$\int_{0}^{T}\left|v_{q}(\sigma)\right| d \sigma$ & 923 & 1600 & 6786 & & 768 & 2210 & 6265 \\
\hline
\end{tabular}


of both controllers given in Figure A2. The control inputs $v_{d}(t)$ and $v_{q}(t)$ are displayed in Figures A3 and A4, respectively. The absolute values of the tracking error $e(t)$ and the control inputs $v_{d}(t)$ and $v_{q}(t)$ and their integrals at three predefined instances where the reference velocity profile in Equation (50) settles to its desired maximum velocity (at $T=12.7 \mathrm{sec}$ ), the midpoint (at $T=22.9 \mathrm{sec}$ ) and at the end of the pattern (at $T=36 \mathrm{sec}$ ) are presented in Table 1. As illustrated by Figures A3 and A4 and Table 1, the proposed backstepping controller performs better tracking with relatively smaller control energy.

The second set of simulations were then performed using the more realistic desired velocity profile in Equation (50) with the same control gains obtained for the first simulations. The results of the second simulations are presented in Figures A5-A8 and Table 2. It is clear that the proposed robust method achieved better tracking with a low-energy profile, although it did not require the exact knowledge of the overall system parameters.

\section{Conclusions}

In this paper, a robust backstepping controller scheme for variable-speed wind turbines with PMSGs was presented. Via the use of a high-gain continuous robust controller in conjunction with backstepping, the proposed method achieved practical tracking of the generator velocity error despite the presence of parametric uncertainty on both the mechanical and the electrical subsystems, that is, the generator velocity tracking error can be driven uniformly to an adjustable small bound even when the dynamical system parameters required for most other previously proposed controller implementations are not available. The boundedness of the system states and the stability of the closed-loop system were guaranteed via Lyapunov-based techniques. Comparative simulation studies were presented to illustrate the performance, feasibility and effectiveness of the proposed method.

\section{Note}

1. A preliminary version of this work appeared in Seker, Zergeroglu, and Tatlicioglu (2012).

\section{Notes on contributors}

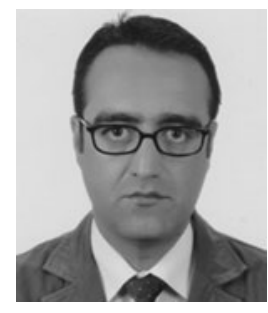

Murat Şeker received the BSc degree from Yildiz Technical University in 1994 and the MSc degree from Gebze Institute of Technology in 1998, both in electrical engineering. Currently, he is pursuing the $\mathrm{PhD}$ degree at the Gebze Institute of Technology, where he also holds a teaching position. His research interests include non-linear control applications, power electronic systems and electromagnetic systems.

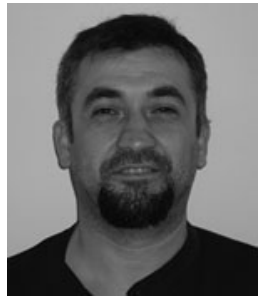

Erkan Zergeroğlu received the BSc and MSc degrees in electrical and electronics engineering from Hacettepe University (Ankara, Turkey) and Middle East Technical University (Ankara, Turkey) in 1992 and 1996, respectively. In 2000, he received the $\mathrm{PhD}$ degree in electrical and computer engineering from Clemson University, Clemson, SC, USA. From 2000 to 2002, he worked for Lucent Technologies-Bell Labs Innovations, Optical Fiber Solutions Sturbridge MA, as a member of technical staff. In 2002, he joined the Computer Engineering Department of Gebze Institute of Technology, Kocaeli, Turkey. His main research interests are in the fields of non-linear-based robust, adaptive and learning control with application to electromechanical systems including robot manipulators, visual servoing and wind turbines.

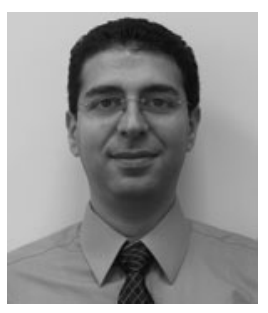

Enver Tatlicioğlu received the BSc degree in electrical and electronics engineering from Dokuz Eylul University, Izmir, Turkey and the $\mathrm{PhD}$ degree in electrical and computer engineering from Clemson University, Clemson, SC, USA in 1999 and 2007, respectively. Upon completion of his $\mathrm{PhD}$ degree, he worked as a post-doctoral research fellow in the Department of Electrical and Computer Engineering at Clemson University, then he joined the Department of Electrical and Electronics Engineering at Izmir Institute of Technology, Izmir, Turkey where he is currently an associate professor. His research interests include control and identification of time delay systems, dynamic modeling of extensible continuum robot manipulators, non-linear control techniques for kinematically redundant robot manipulators, partial-state feedback and output feedback control, haptic systems and teleoperation and learning, robust and adaptive control of non-linear systems.

\section{References}

Abdin, E., and Xu, W. (2000), 'Control Design and Dynamic Performance Analysis of a Wind Turbine-Induction Generator Unit', IEEE Transactions on Energy Conversion, 15(1), 91-96.

Abedini, A., and Nikkhajoei, H. (2011), 'Dynamic Model and Control of a Wind- Turbine Generator With Energy Storage', IET Renewable Power Generation, 5, $67-78$.

Beltran, B., Ahmed-Ali, T., and Hachemi Benbouzid, M.E. (2008), 'Sliding Mode Power Control of Variable-Speed Wind Energy Conversion Systems', IEEE Transactions on Energy Conversion, 23(2), 943-948.

Beltran, B., Ahmed-Ali, T., and Hachemi Benbouzid, M.E. (2009), 'High-Order Sliding-Mode Control of Variable-Speed Wind Turbines', IEEE Transactions on Industrial Electronics, 56(9), 3314-3321.

Boukhezzar, B., and Siguerdidjane, H. (2005), 'Nonlinear Control of Wind Turbines for Power Regulation', in Proceedings of IEEE International Conference on Control Applications, Toronto, Canada, pp. 114-119.

Dawson, D., Bridges, M., and Qu, Z. (1995), Nonlinear Control of Robotic Systems for Environmental Waste and Restoration, Englewood Cliffs, NJ: Prentice Hall.

Dawson, D., Hu, J., and Burg, T. (1998), Nonlinear Control of Electric Machinery, New York: Marcel Dekker. 
Ferreira, A., Bejarano, F., and Fridman, L. (2012), 'Unmatched Uncertainties Compensation Based on High-Order Sliding Mode Observation', International Journal of Robust and Nonlinear Control, 23(7), 754-765.

Galdi, V., Piccolo, A., and Siano, P. (2008), 'Designing an Adaptive Fuzzy Controller for Maximum Wind Energy Extraction', IEEE Transactions on Energy Conversion, 23(2), 559569.

Iyasere, E., Salah, M., Dawson, D., and Wagner, J. (2008), 'Nonlinear Robust Control to Maximize Energy Capture in a Variable Speed Wind Turbine', in Proceedings of the American Control Conference, Seattle, WA, pp. 1824-1829.

Jagannathan, S., and Lewis, F.L. (2000), 'Robust Backstepping Control of a Class of Nonlinear Systems Using Fuzzy Logic', Information Sciences, 123(3-4), 223-240.

Kwan, C., and Lewis, F.L. (2000), 'Robust Backstepping Control of Nonlinear Systems Using Neural Networks', IEEE Transactions on Systems, Man, and Cybernetics-Part A: Systems and Humans, 30(6), 753-766.

Maureen, H.M., and Balas, M.J. (2002), 'Systematic Control Design Methodology for Variable Speed Wind Turbine', Technical Report NREL/TP-500-29415, National Renewable Energy Laboratory, Golden, CO.

Morfin, O.A., Loukianov, A., Canedo, J.M., and Castellanos M.I. (2010), 'Velocity Controller of a Wound Rotor Induction Generator via Block Control Linearization via Second Order Sliding Modes', in 7th International Conference on Electrical Engineering, Computing Science and Automatic Control (CCE 2010), Chiapas, Mexico, pp. 77-82.

Muldaji, E., Pierce, K., and Migliore, P. (1998), 'Control Strategy for Variable-Speed, Stall-Regulated Wind Turbines', in Proceedings of the American Control Conference, Philadelphia, PA, pp. 1710-1714.

Müller, S., Deicke, M., and De Doncker, R.W. (2002), 'Doubly Fed Induction Generator System for Wind Turbines', IEEE Industry Applications Magazine, 8(3), 26-33.

Oguz, Y., and Guney, I. (2010), 'Adaptive Neuro-Fuzzy Inference System to Improve the Power Quality of VariableSpeed Wind Power Generation System', Turkish Journal of Electrical Engineering and Computer Sciences, 18(4), 625645.
Ozbay, U., Zergeroglu, E., and Sivrioglu, S. (2008), 'Adaptive Backstepping Control of Variable Speed Wind Turbines', International Journal of Control, 81, 910-919.

Qu, Z. (1998), Robust Control of Nonlinear Uncertain Systems, Wiley Series in Nonlinear Science, New York: John Wiley and Sons, Inc.

Seker, M., Zergeroglu, E., and Tatlicioglu, E. (2012), 'Robust Backstepping Control of Variable Speed Wind Turbines With Permanent Magnet Synchronous Generators', in IEEE MultiConference on Systems and Control, Dubrovnik, Croatia, pp. 1068-1073.

Senjyu, T., Sakamoto, R., Urasaki, N., Funabashi, T., Fujita, H., and Sekine, H. (2006), 'Output Power Leveling of Wind Turbine Generator for All Operating Regions by Pitch Angle Control', IEEE Transactions on Energy Conversion, 21(2), 467-475.

Sivrioglu, S., Ozbay, U., and Zergeroglu, E. (2008), 'Variable Speed Control of Wind Turbines: A Robust Backstepping Approach', in Proceedings of the 17th World Congress The International Federation of Automatic Control, Seoul, Korea, pp. 1183-1188.

Song, Y., Dhinarakaran, B., and Bao, X. (2000), 'Variable Speed Control of Wind Turbines Using Nonlinear and Adaptive Algorithms', Journal of Wind Engineering and Industrial Aerodynamics, 85, 293-308.

\section{Appendix A. Dynamic terms}

The linearly parameterisable terms in Equation (20) are explicitly defined in the following form:

$$
\begin{aligned}
W_{1} & \triangleq\left[\frac{\widehat{J}}{\widehat{\phi}_{m}} \ddot{w}_{d} \frac{\widehat{B P}}{2 \widehat{\phi}_{m}} \dot{w}_{d} \frac{K}{\widehat{\phi}_{m}} \dot{w}_{d} \frac{K}{\widehat{\phi}_{m}} w_{g} \frac{K}{\widehat{\phi}_{m}} i_{d} i_{d} i_{q} w_{q} w_{g}\right],( \\
\theta_{1} & \triangleq\left[-L_{d}-L_{d}-L_{d}-L_{d} \frac{B P}{2 J}-L_{d} \frac{\phi_{m}}{J} R_{s} L_{q} k_{g} \lambda_{m}\right]^{T},
\end{aligned}
$$

where the auxiliary time-varying term $K$ contains known variables and is defined as

$$
K \triangleq k_{e}+2 k_{n} \rho_{1} e \frac{\partial}{\partial e} \rho_{1}+k_{n} \rho_{1}^{2}+\frac{\partial}{\partial e} v_{R 1}
$$



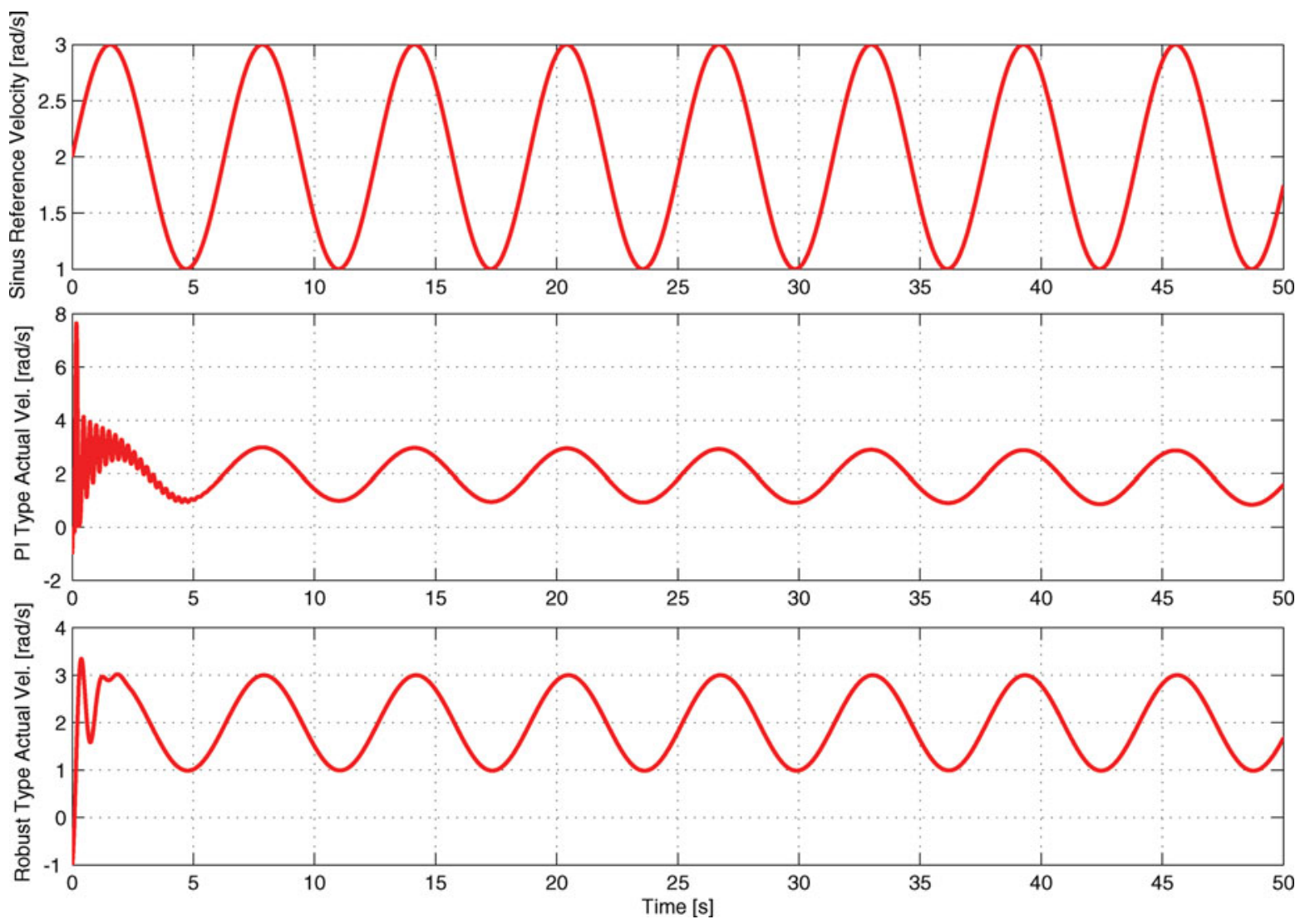

Figure A1. First simulation - reference generator velocity profiles (top), actual generator velocity profile of PI controller (middle) and the robust controller (bottom).
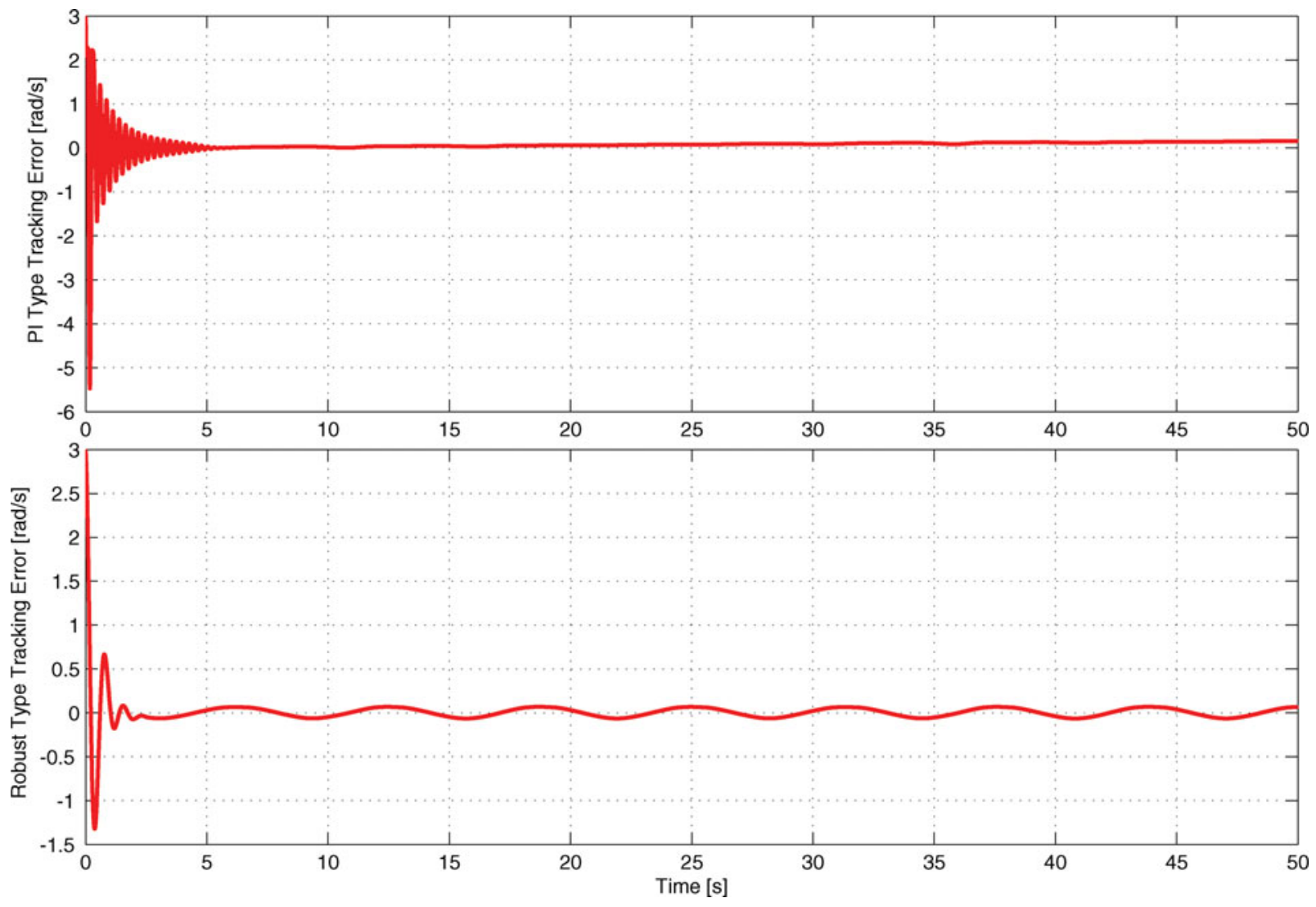

Figure A2. First simulation - velocity tracking error signals, $e(t)$, for PI- (top) and robust-type controllers (bottom). 

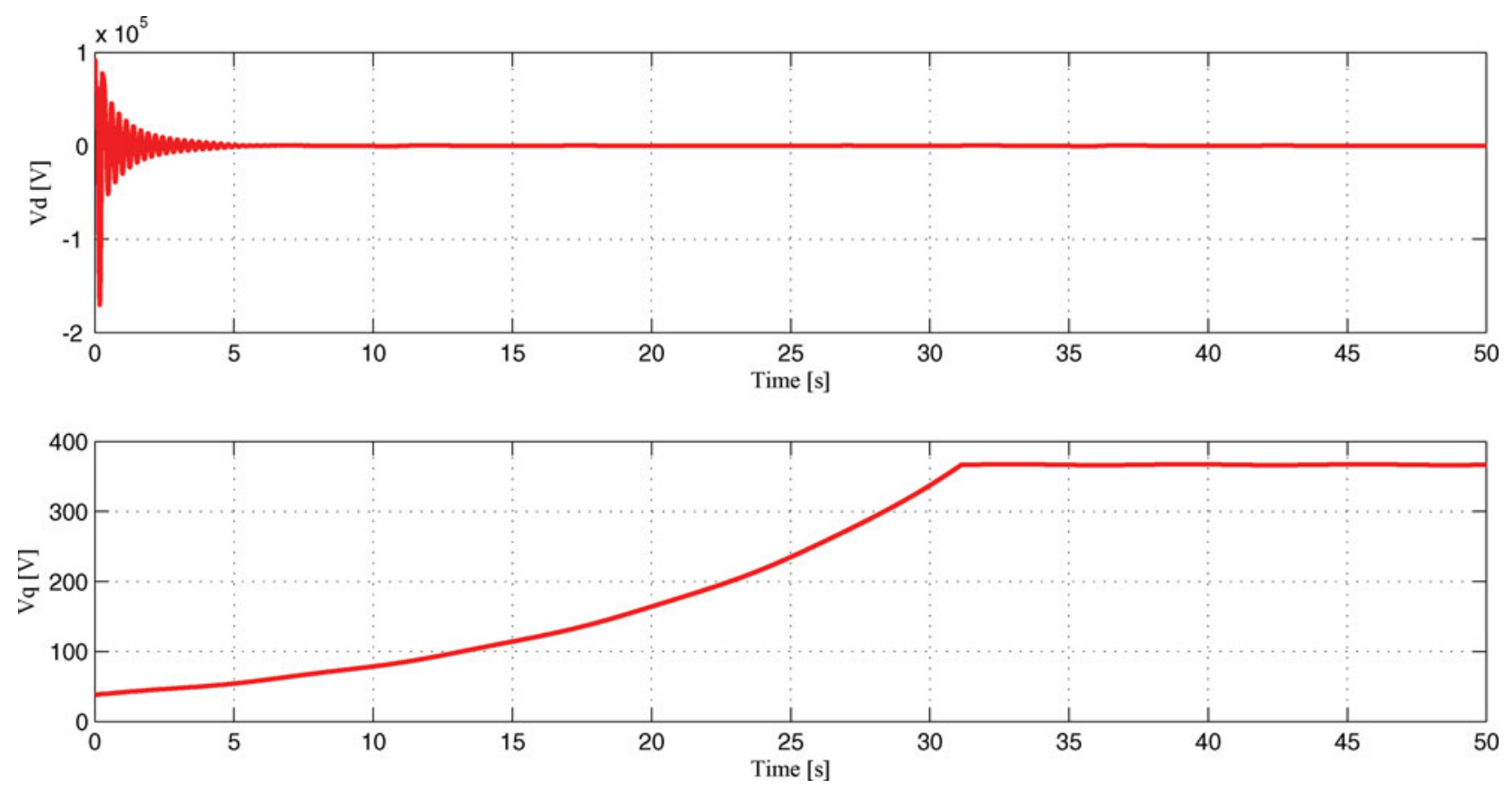

Figure A3. First simulation - PI-type controller: voltages applied to the generator, $v_{d}(t)$ (top) and $v_{q}(t)$ (bottom).
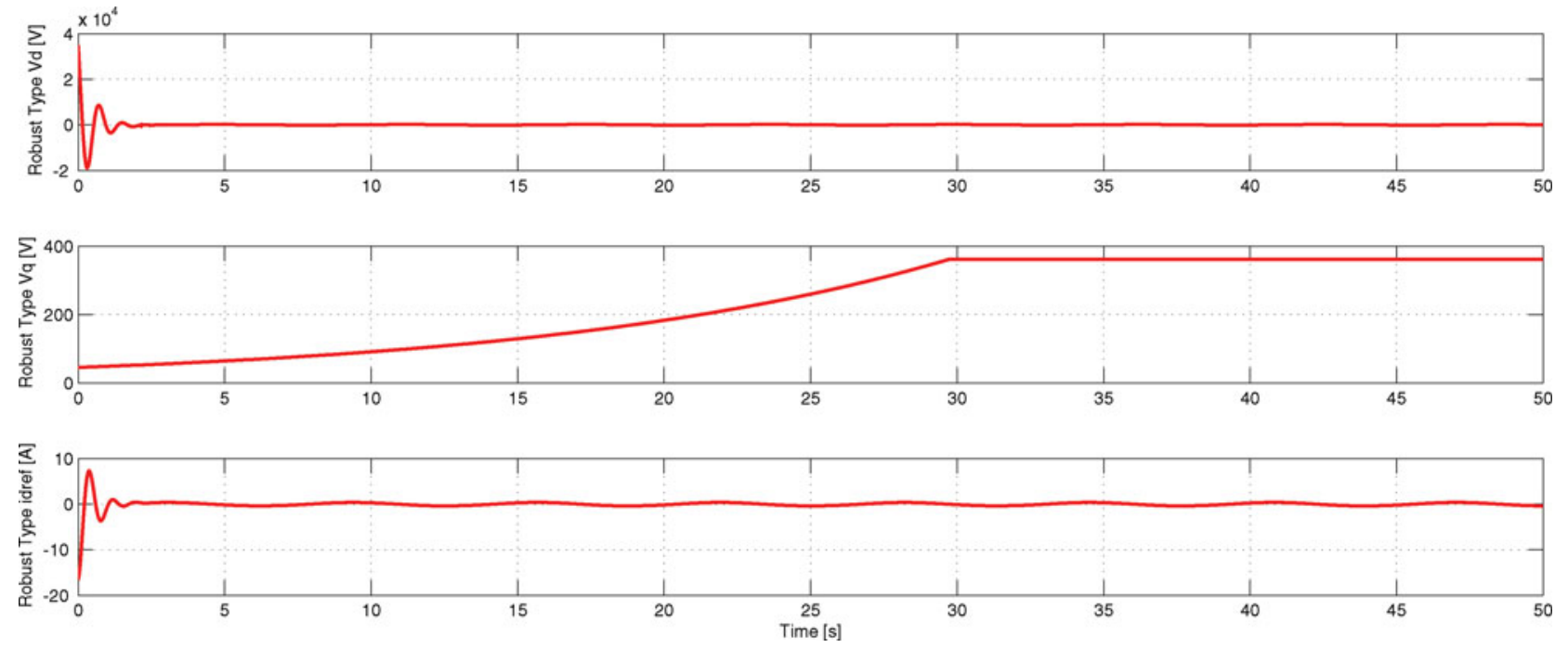

Figure A4. First simulation - robust backstepping controller: voltages applied to the generator, $v_{d}(t)$ (top), $v_{q}(t)$ (middle) and the auxiliary control input $i_{d \text { ref }}$ (bottom). 

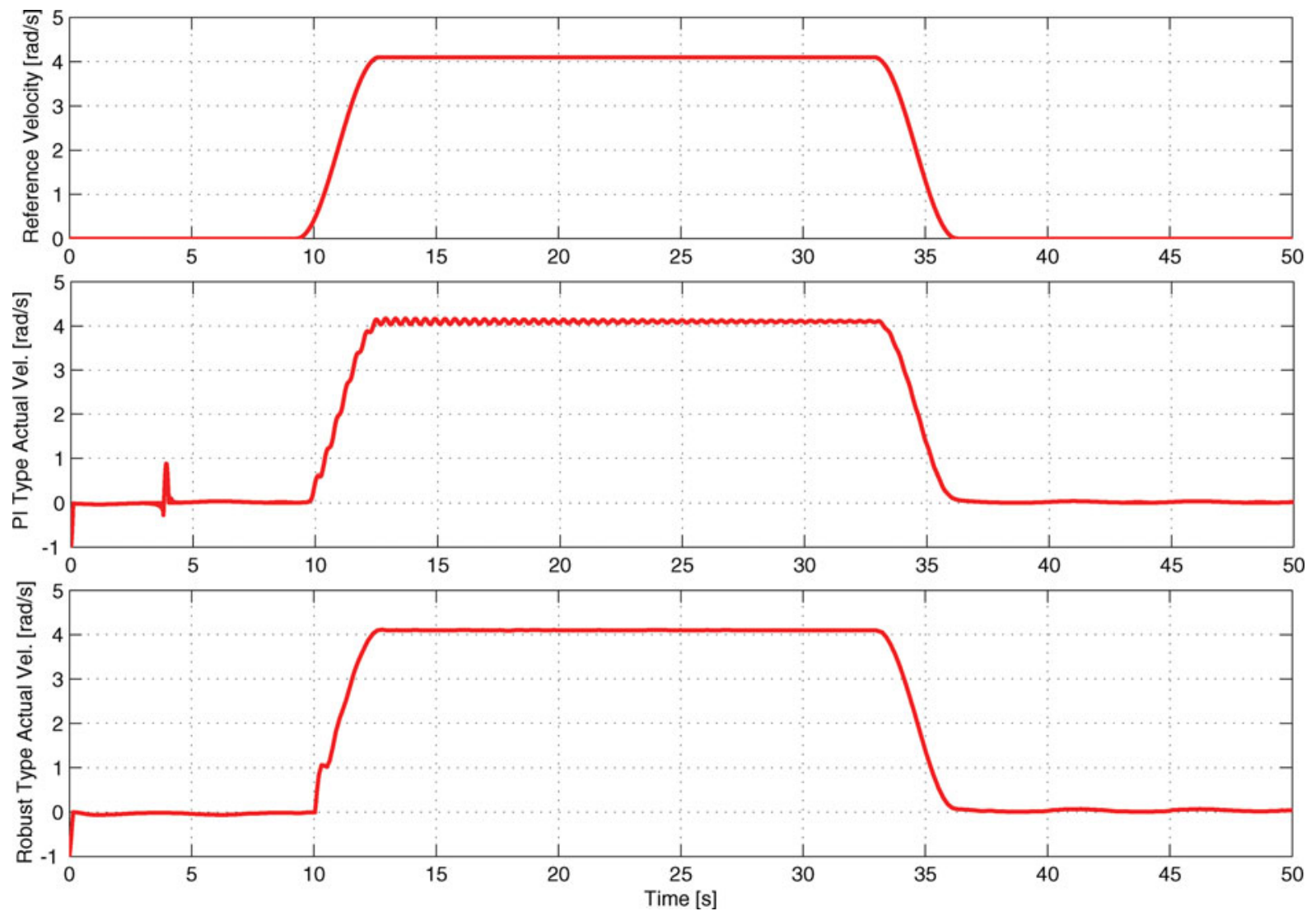

Figure A5. Second simulation - reference generator velocity profiles (top), actual generator velocity profile of PI controller (middle) and the robust controller (bottom).
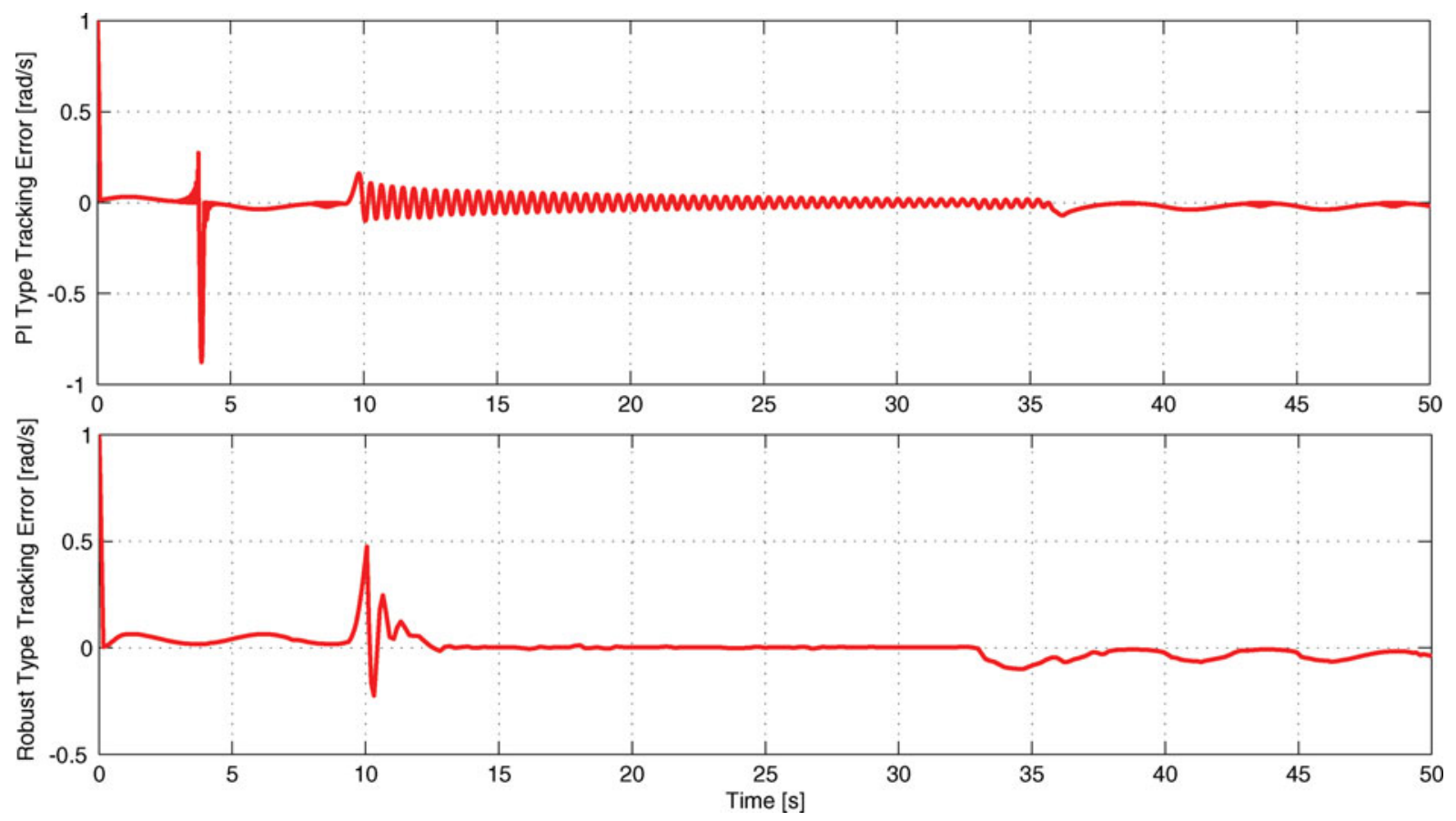

Figure A6. Second simulation - velocity tracking error signals, $e(t)$, for PI- (top) and robust-type controllers (bottom). 

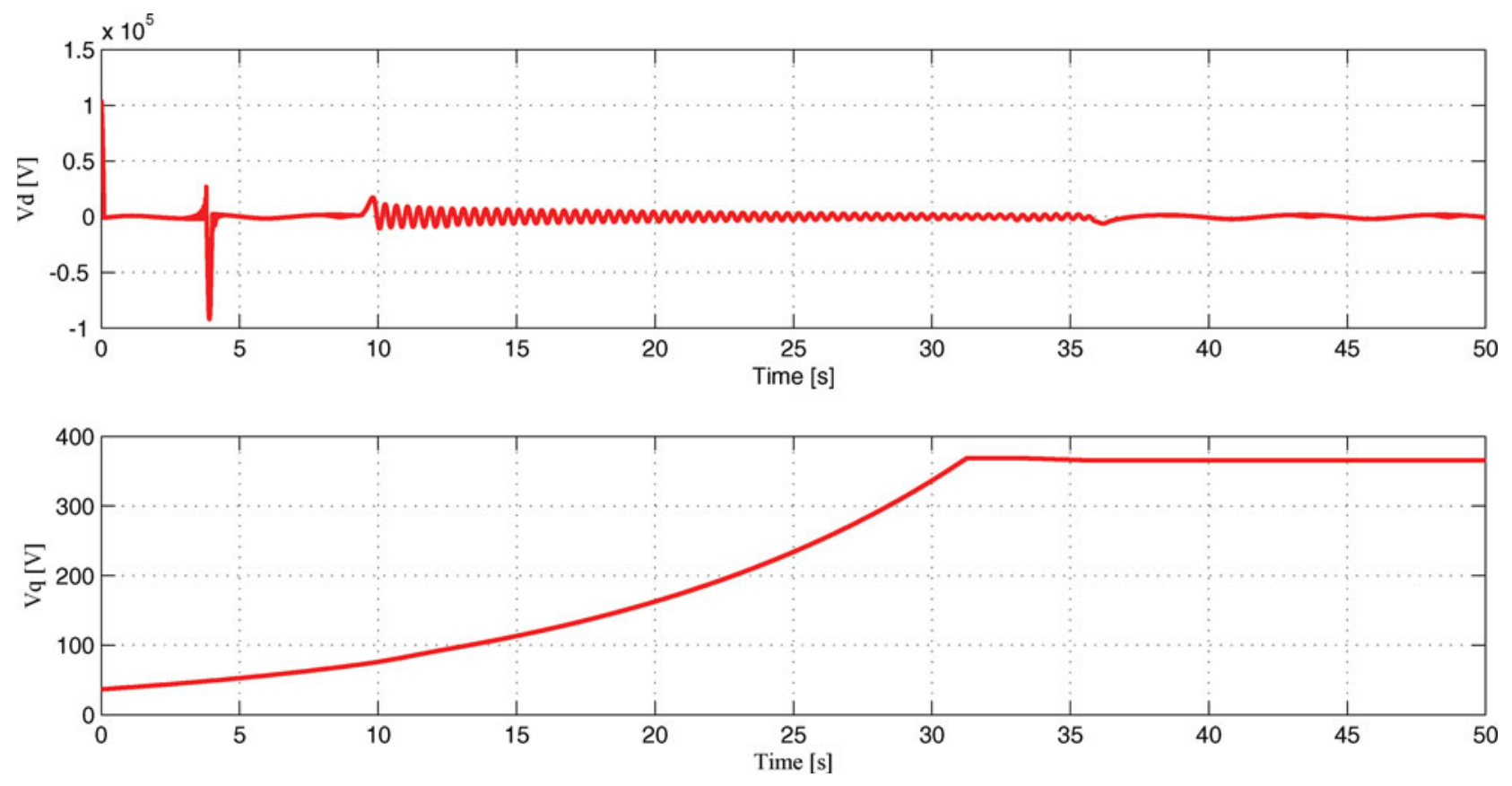

Figure A7. Second simulation - PI-type controller: voltages applied to the generator, $v_{d}(t)$ (top) and $v_{q}(t)$ (bottom).
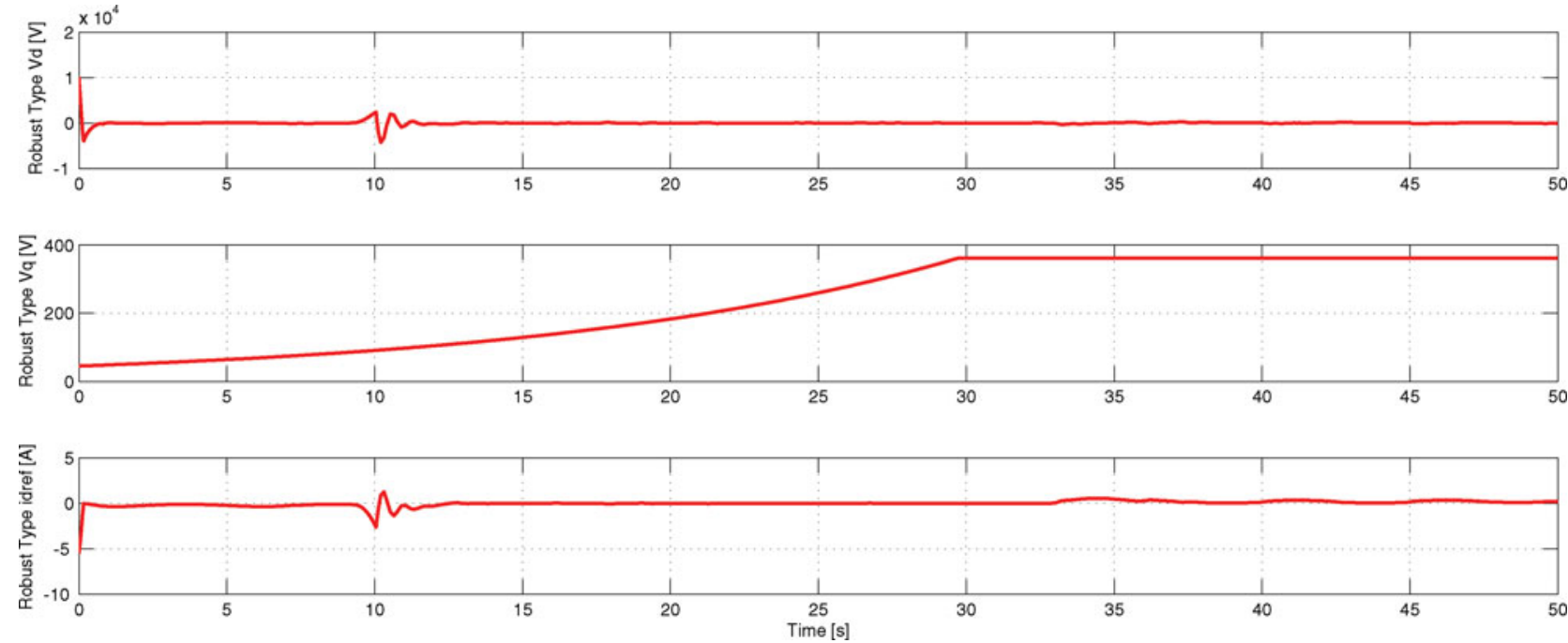

Figure A8. Second simulation - robust backstepping controller: voltages applied to the generator, $v_{d}(t)$ (top), $v_{q}(t)$ (middle) and the auxiliary control input $i_{d \text { ref }}$ (bottom). 